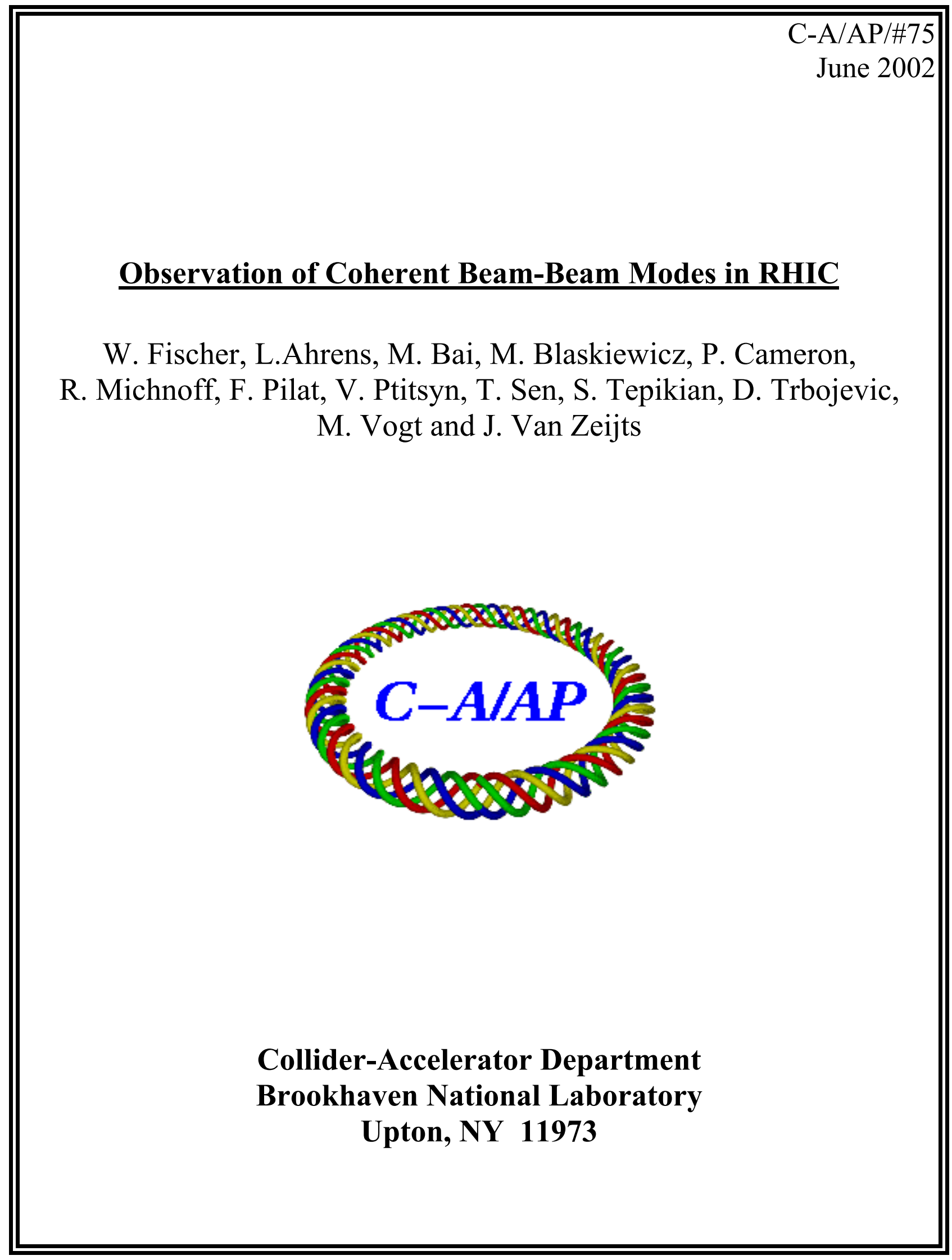


BNL C-AD/AP/75

\title{
Observation of Coherent Beam-Beam Modes in RHIC
}

\author{
W. Fischer, L. Ahrens, M. Bai, M. Blaskiewicz, P. Cameron, \\ R. Michnoff, F. Pilat, V. Ptitsyn, T. Sen*, S. Tepikian, \\ D. Trbojevic, M. Vogt $^{\dagger}$, and J. v. Zeijts
}

June 21, 2002

\begin{abstract}
Colliding beams with equal intensities and close tunes in either plane can give rise to coherent beam-beam modes. If the beam-beam interaction is the dominant source for transverse nonlinearities, the frequencies of the coherent modes can be outside the continuous frequency spectrum, and are therefore not Landau damped. Coherent beam-beam modes were observed in RHIC in a dedicated experiment and in routine operation. We describe the machine and beam conditions that gave rise to these modes.
\end{abstract}

\section{Introduction}

The beam-beam interaction can give rise to coherent transverse dipole oscillation modes. In the simplest case with only one collision per turn there are two modes per transverse plane. The $\sigma$-mode frequency is the same as the betatron frequency without beam-beam interaction, and the $\pi$-mode frequency is shifted downwards for particles of the same charge. Alexahin pointed out that the $\pi$-mode may not be Landau damped if the beambeam interaction is the dominant source for tune spread and the beam intensities are approximately the same [1]. The tune spread from the beam-beam interactions is the beam-beam parameter $\xi$ while the the $\pi$ mode is shifted by $Y \xi$, where $Y \approx 1.3$ is the Yokoya factor $[2,3]$. Thus the $\pi$-mode frequency can be outside the continuous frequency spectrum.

Coupled coherent beam-beam oscillations were already observed in the CERN ISR with unbunched beams and currents of about $30 \mathrm{~A}$ [4-7]. With a beam-beam parameter of $\xi=0.003$ beams collided at up to eight points in the ring. The beam-beam interaction modified the threshold of the resistive wall instability, leading to beam losses, typically on a time scale of seconds. Beams with reduced chromaticity, and therefore less tune spread, were found to be more susceptible to the instability.

RHIC is the only existing collider with hadron beams of equal intensities and therefore the only existing hadron machine where $\pi$-modes can be expected. Coherent beam-beam modes may also be relevant in future hadron colliders like the LHC and VLHC. $\sigma$ - and $\pi$-modes were created in a RHIC experiment, and were also observed in routine operation

\footnotetext{
*Fermi National Accelerator Laboratory

${ }^{\dagger}$ University of New Mexico
} 
Table 1: RHIC parameters for the coherent beam-beam mode experiment.

\begin{tabular}{|c|c|c|c|}
\hline quantity & symbol & Blue & Yellow \\
\hline ion species & $\ldots$ & $\mathrm{p}^{+}$ & $\mathrm{p}^{+}$ \\
\hline relativistic parameter & $\gamma$ & 25.9 & 25.9 \\
\hline number of bunches & $n_{b}$ & 1 & 1 \\
\hline number of collisions per turn & & & \\
\hline transverse tunes & $\left(Q_{x}, Q_{y}\right)$ & $(0.2129,0.2412)$ & $(0.2126,0.2392)$ \\
\hline linear coupling & $\left|Q_{x}-Q_{y}\right|_{\min }$ & 0.011 & 0.013 \\
\hline $\begin{array}{l}\text { chromaticities } \\
\text { synchrotron tune }\end{array}$ & $\begin{array}{c}\left(\xi_{x}, \xi_{y}\right) \\
Q_{s}\end{array}$ & $\begin{array}{c}(2,2) \\
3.8 \cdot 10^{-4}\end{array}$ & $\begin{array}{c}(3,3) \\
3.8 \cdot 10^{-4}\end{array}$ \\
\hline particles per bunch & $N_{b}$ & $0.84 \cdot 10^{11}$ & $0.88 \cdot 10^{11}$ \\
\hline normalized emittance (95\%) & $\epsilon_{x, y}$ & $20 \mu \mathrm{m}$ & $20 \mu \mathrm{m}$ \\
\hline beam-beam parameter & $\xi$ & \multicolumn{2}{|c|}{0.003} \\
\hline
\end{tabular}

with protons. With a beam-beam parameter $\xi$ of up to 0.0025 in proton operation and four collisions, coherent modes have not limited the collider operation so far. However, it is expected that the beam-beam parameter can be increased by a factor of 3 in the future and coherent beam-beam modes may become a concern.

\section{Coherent Beam-Beam Modes in the Experiment}

The beam-beam experiment was aimed at creating and observing coherent beam-beam modes. It was carried out with protons at the injection energy of $23.4 \mathrm{GeV}$, to allow for fast refills. In the AGS injector the intensity per bunch was maximized and the emittance minimized to obtain the largest possible beam-beam parameter. RHIC was prepared by checking and correcting the closed orbit, the injection conditions and the chromaticities $\left(\xi_{x}, \xi_{y}\right)$. A relatively large linear coupling remained. The experimental conditions are summarized in Tab. 1.

In addition to the $28 \mathrm{MHz}$ accelerating rf system, a $197 \mathrm{MHz}$ Landau cavity was used in each ring to suppress coherent longitudinal oscillations, which are otherwise present for long periods of time. With $150 \mathrm{kV}$ gap voltage from the accelerating system, the voltage of the $197 \mathrm{MHz}$ cavity was raised to $60 \mathrm{kV}$ after a bunch was injected, and then lowered to $10 \mathrm{kV}$. The resulting longitudinal profiles are shown in Fig. 1. Coherent longitudinal oscillations are likely to disturb the transverse tune measurement and transfer function measurements with the phased locked loop (PLL) system. The PLL tune measurement system has a resolution of better than $10^{-5}$.

Collisions were set up at the 10 o'clock interaction point (IP) only, using orbit monitors, collision signals, and the beam-beam tune shift. At all other interaction points the beams were separated vertically by at least $6 \mathrm{rms}$ beam sizes. The evolution of the beam intensities under collision conditions is shown in Fig. 2. In Fig. 3 the Phobos collision signal and both transverse tunes of both rings are depicted during the tuning process. 

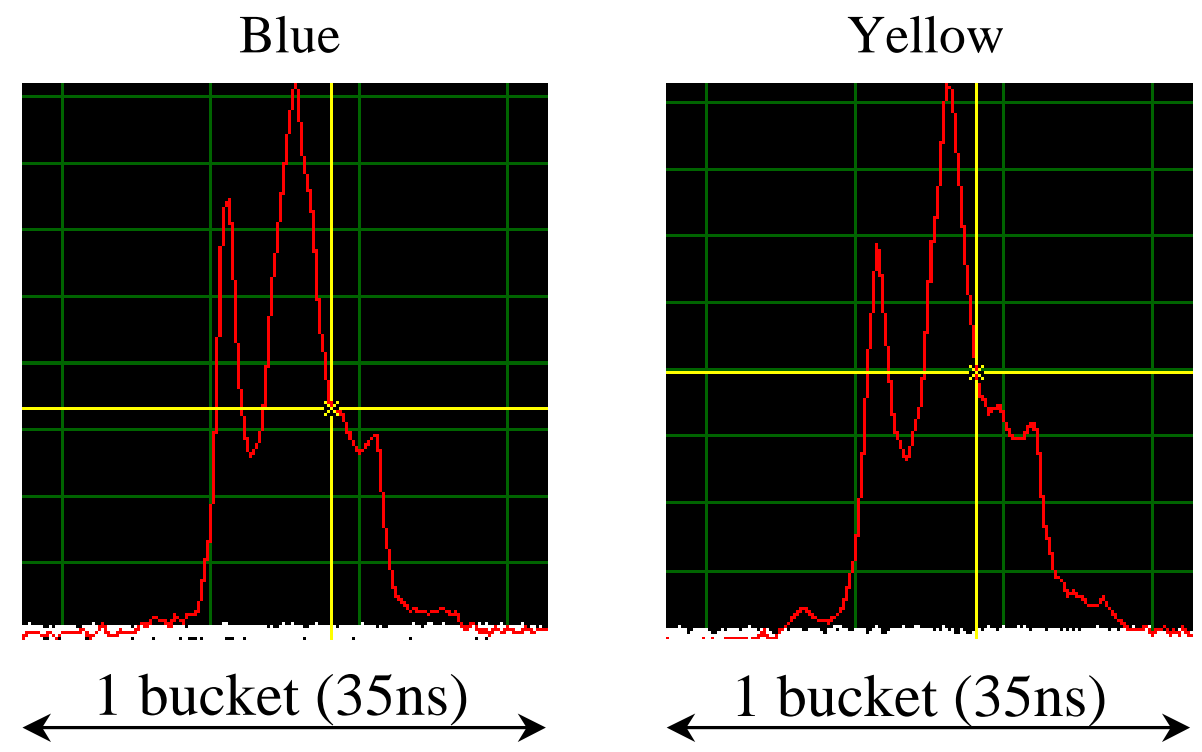

Figure 1: Blue and Yellow longitudinal beam profiles during the experiment.

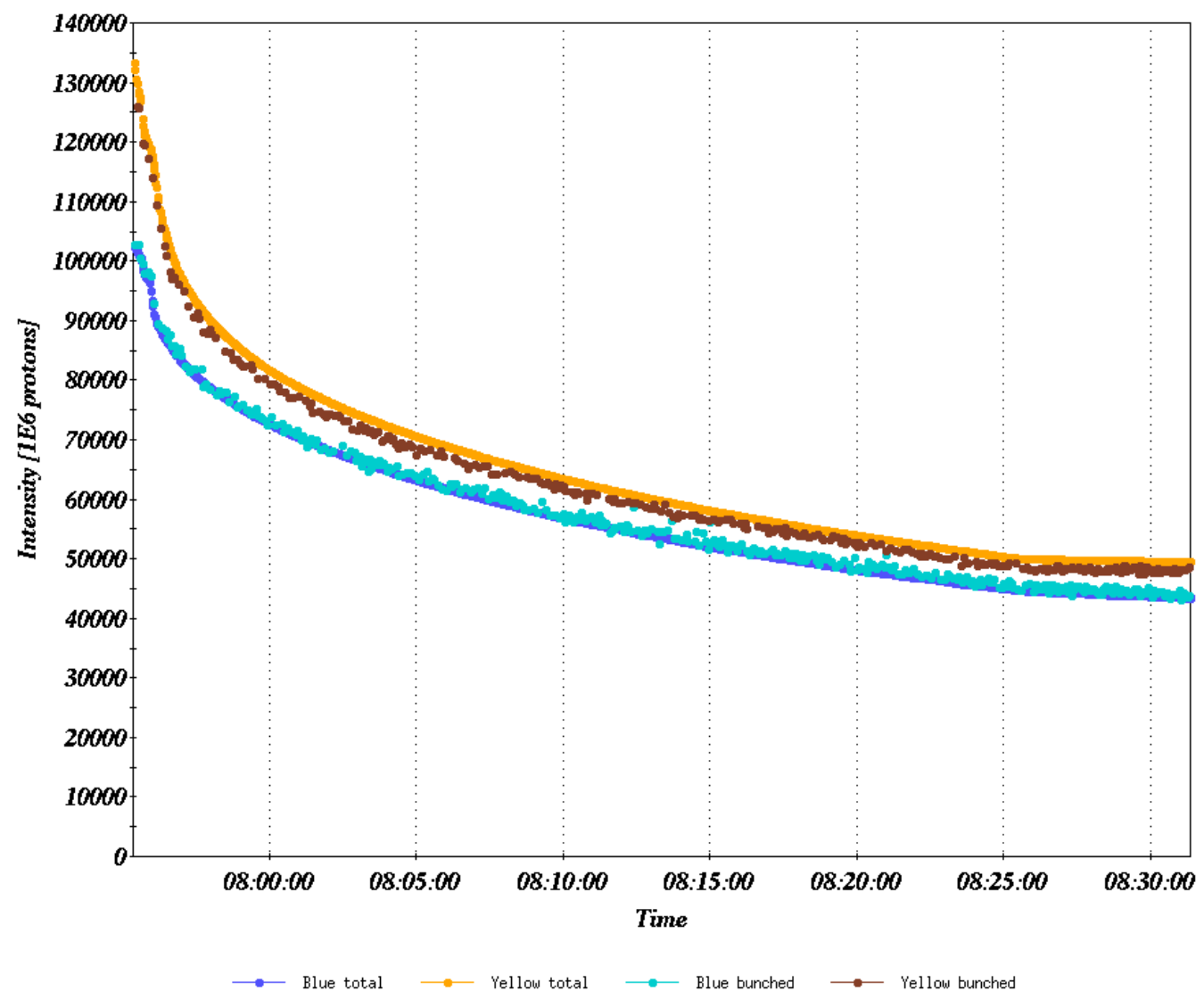

Figure 2: Blue and Yellow beam intensities in the experiment with colliding beams. Shown are both the total and bunched intensities for each ring. 
Phobos collision signal
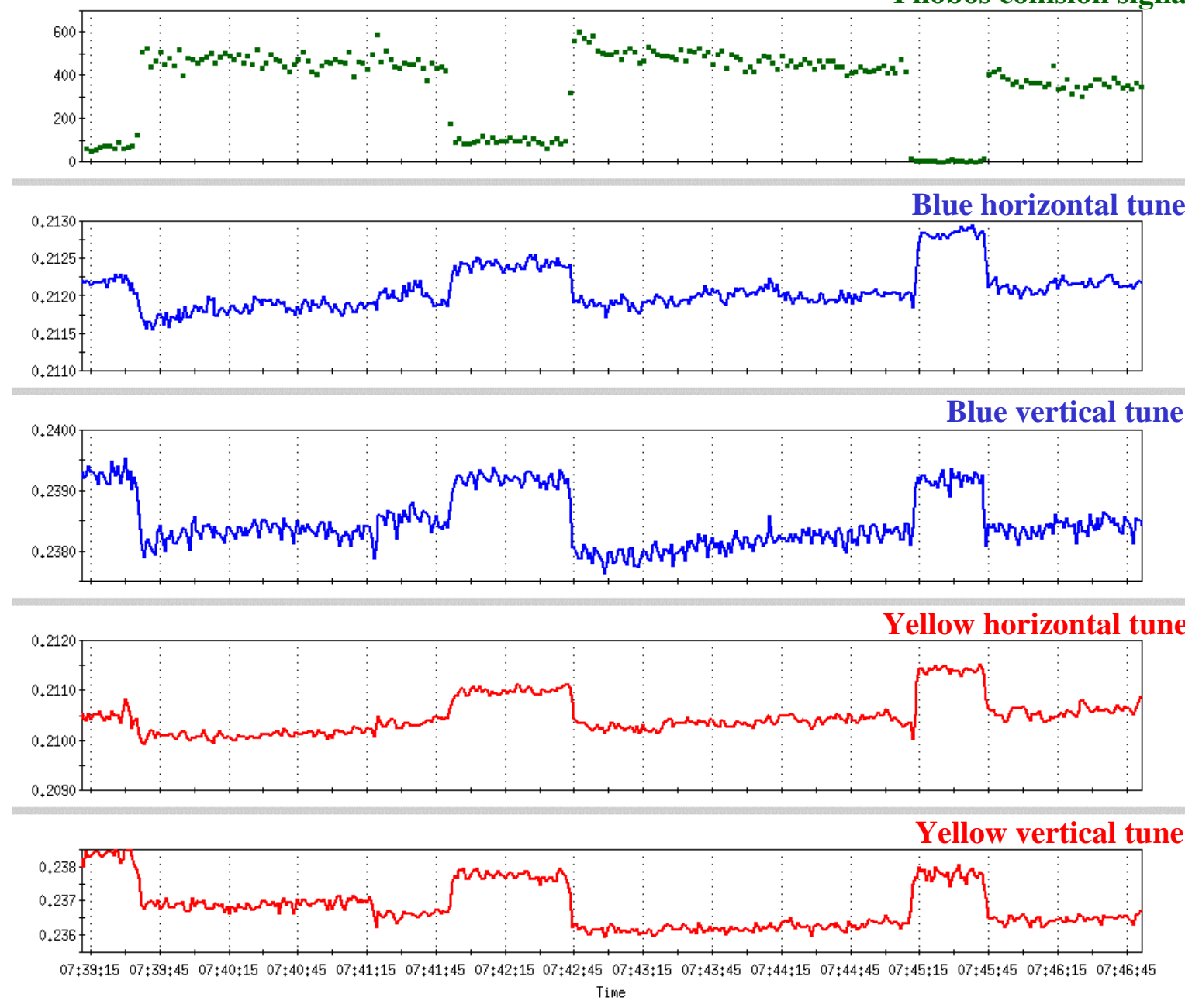

Figure 3: Phobos collision signal and Blue and Yellow horizontal and vertical tunes with and without beam-beam interaction. With collisions, the Phobos collision signal rises and all tunes are depressed.

When the beams are in collision, the Phobos collision signal rises and all tunes are depressed from the beam-beam interaction. When the beams were separated longitudinally, the Phobos collision signal disappears and the tunes increase again. The measured tune change from the beam-beam interaction of approximately 0.0015 is half the beam-beam parameter $\xi$. The tunes in both rings were set at almost the same horizontal and vertical tunes $\left(Q_{x}, Q_{y}\right)$.

For the measurement of coherent modes, a single bunch of protons was filled in each ring. Transverse spectra were obtained from up to 4096 turns recorded in a beam position monitor after the blue beam experienced a small kick by the tune kicker, corresponding to about one tenth of an rms beam size. In all cases the beams decohered in about 1000 turns after the kick. The beam-beam interaction can be switched on and off by separating the beams longitudinally at the IP. 
Blue Horizontal, single p bunch, at injection

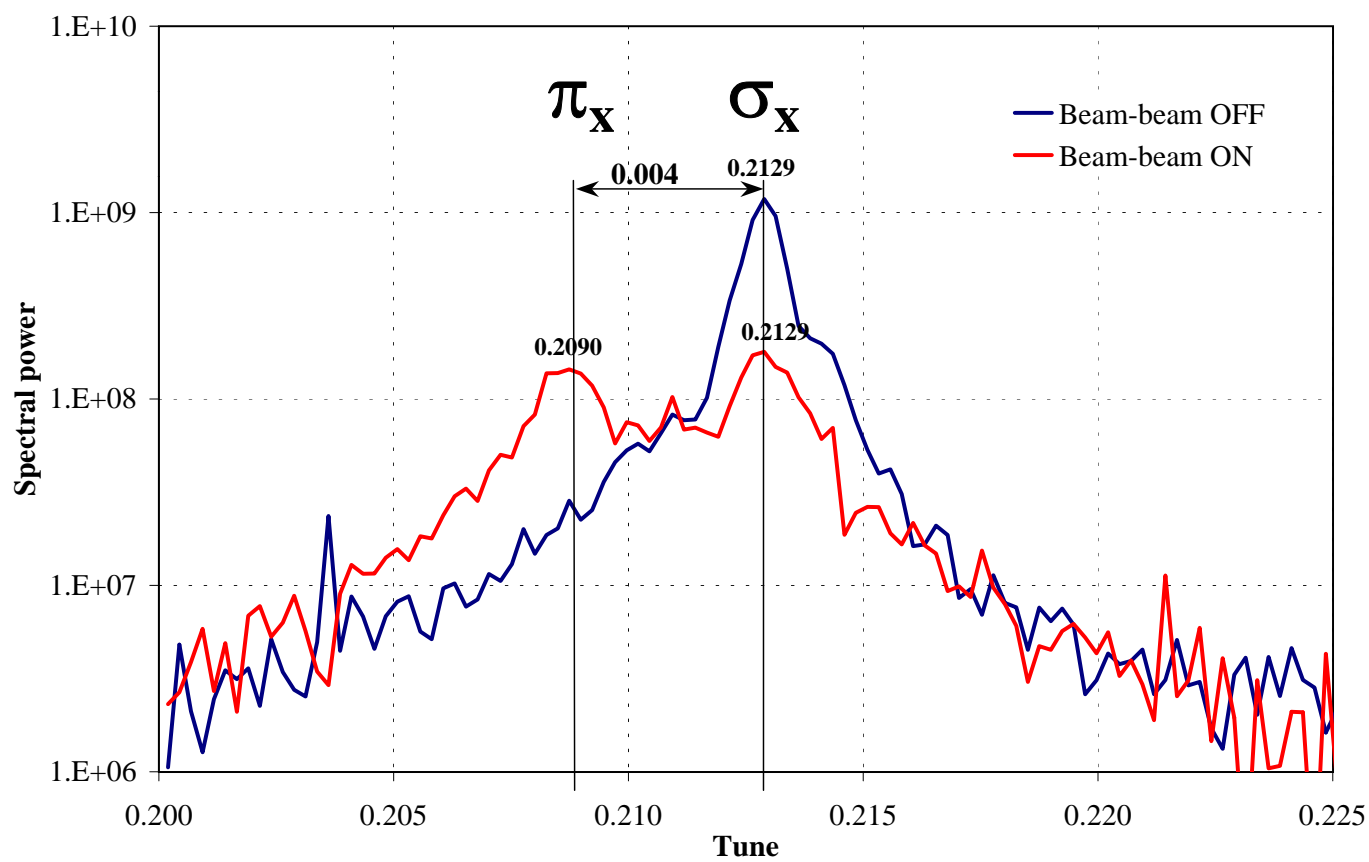

Figure 4: Blue horizontal spectra from 4096 consecutive turns after a small kick in the experiment. The two curves show the situation with beam-beam interaction on and off.

Yellow Horizontal, single $p$ bunch, at injection

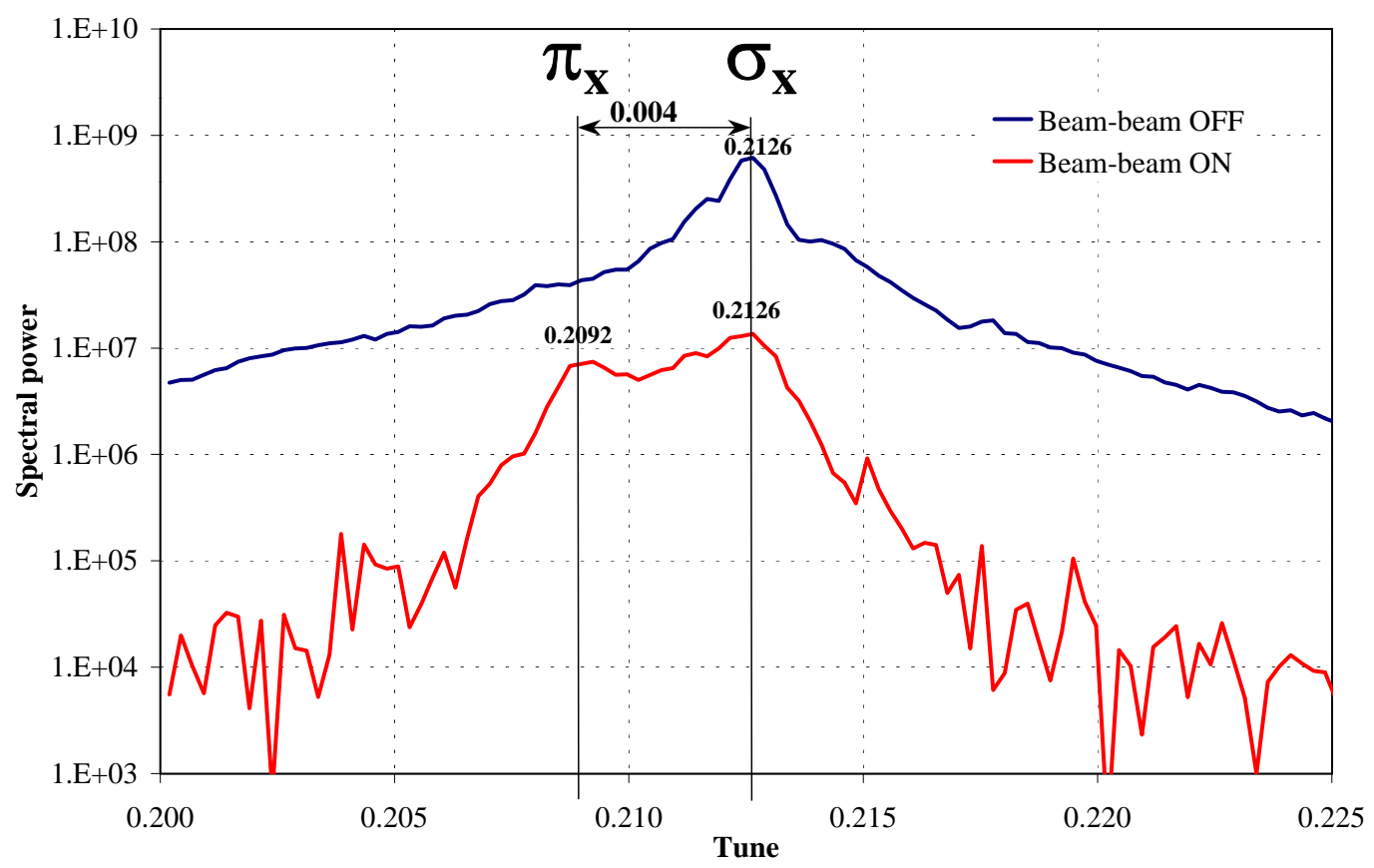

Figure 5: Yellow horizontal spectra from 4096 consecutive turns after a small kick in the experiment. The two curves show the situation with beam-beam interaction on and off. 
Table 2: RHIC parameters for both beams when coherent modes were observed in operation.

\begin{tabular}{lccc}
\hline \hline quantity & symbol & Blue & Yellow \\
\hline ion species & $\ldots$ & $\mathrm{p}^{+}$ & $\mathrm{p}^{+}$ \\
relativistic parameter & $\gamma$ & 106.6 & 106.6 \\
number of bunches & $n_{b}$ & 55 & 55 \\
number of collisions per turn & $\ldots$ & \multicolumn{2}{c}{4} \\
transverse tunes & $\left(Q_{x}, Q_{y}\right)$ & $(0.208,0.221)$ & $(0.209,0.221)$ \\
linear coupling & $Q_{x}-\left.Q_{y}\right|_{\text {min }}$ & 0.012 & 0.011 \\
chromaticities & $\left(\xi_{x}, \xi_{y}\right)$ & $(1,2)$ & $(3,2)$ \\
synchrotron tune & $Q_{s}$ & $3.5 \cdot 10^{-4}$ & $3.5 \cdot 10^{-4}$ \\
average particles per bunch & $N_{b}$ & $0.72 \cdot 10^{11}$ & $0.69 \cdot 10^{11}$ \\
normalized emittance $(95 \%)$ & $\epsilon_{x, y}$ & $30 \mu \mathrm{m}$ & $30 \mu \mathrm{m}$ \\
beam-beam parameter & $\xi$ & \multicolumn{2}{c}{$\mathbf{0 . 0 0 1 5}$} \\
\hline \hline
\end{tabular}

Figs. 4 and 5 show the horizontal spectra of the Blue and Yellow beams with and without beam-beam interaction. Without beam-beam interaction, the horizontal tunes of both beams are close. Only one peak, corresponding to the $\sigma$-mode, is visible. With beam-beam interaction, a second peak, corresponding to the $\pi$-mode, is clearly visible in both the Blue and Yellow spectra. The $\pi$-mode is down-shifted by 0.004 from the $\sigma$-mode. This is in accordance with a measured beam-beam parameter $\xi$ of 0.003 and a Yokoya factor of about 1.3 .

\section{Coherent Beam-Beam Modes in Operation}

Coherent beam-beam modes were also observed in routine operation at the beginning of a proton store during tuning for beam lifetime. In operation, bunches encounter four headon collisions per turn. The appearance of coherent beam-beam modes can can be seen in Fig. 6, where the horizontal and transverse spectra of the Blue beam are seen with and without beam-beam interaction. Note that the tune resolution is only $10^{-3}$. The spectra were obtained from 1024 consecutive turns after a small kick. The horizontal spectra are shifted upwards for better visibility. The beam parameters are summarized in Tab. 2.

Without beam-beam interaction there are two peaks visible in both planes, corresponding to the two transverse tunes with linear coupling and denoted by $\sigma_{x}$ and $\sigma_{y}$. With beam-beam interaction a $\pi$-mode is created for each $\sigma$-mode, with a tune lower by 0.005 compared to the corresponding $\sigma$-mode. The difference of 0.005 between the $\pi$ and $\sigma$-modes is consistent with a beam-beam parameter $\xi=0.0015$, four collisions, and a Yokoya factor $Y \approx 1.3$.

In Fig. 7 the horizontal and vertical spectra of the Yellow beam are shown with and without beam-beam interaction. In this case too linear coupling is visible, but no $\pi$-modes can be distinguished. This may be due to the limited tune resolution of the spectra that were made from only 1024 turns. In the experiment it was found that the $\pi$-modes are less pronounced in the Yellow beam spectra (compare Fig. 4 with Fig. 5). 
Blue Transverse Spectra

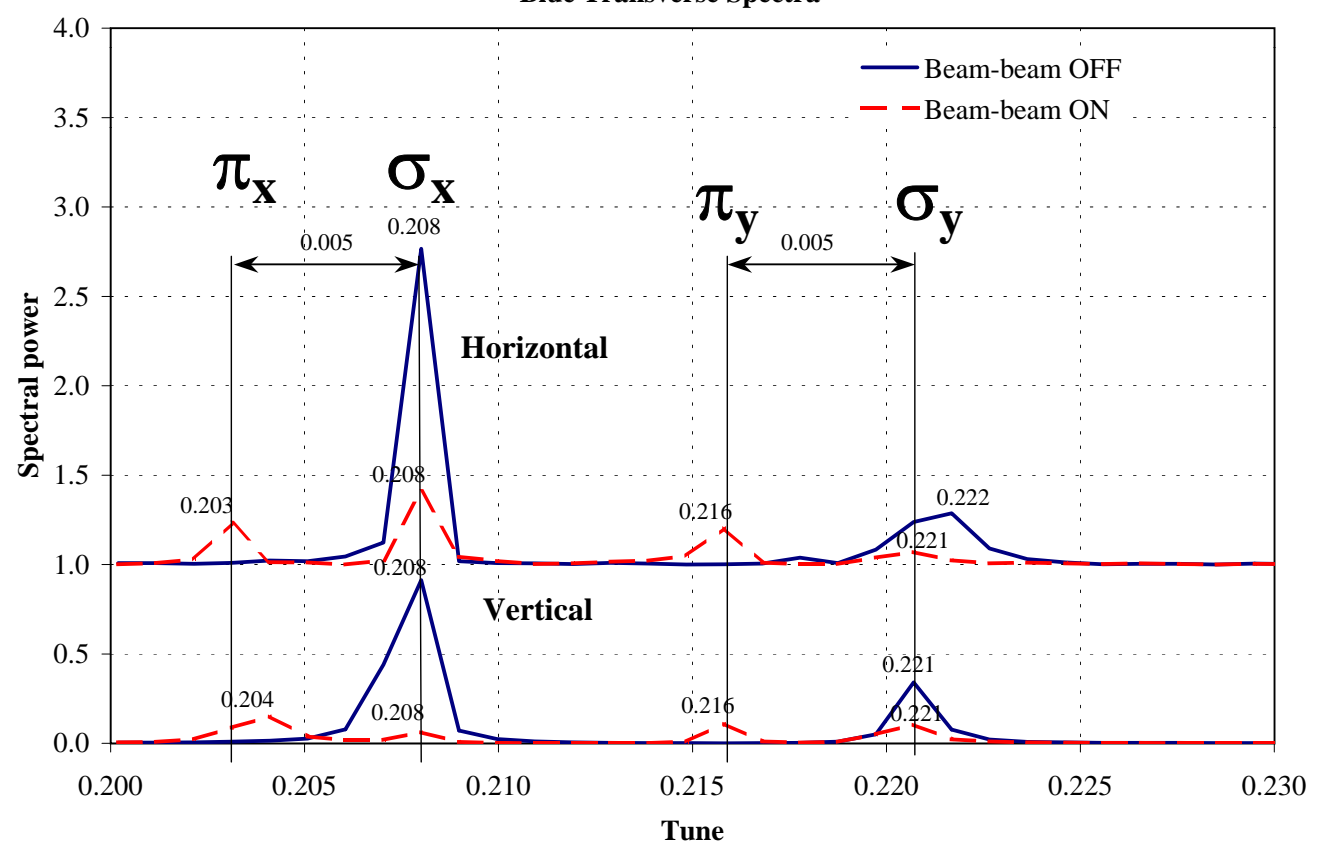

Figure 6: Blue transverse spectra from 1024 consecutive turns after a small kick in operation. Two curves show the situation with the beam-beam interaction on, two with the beam-beam interaction off. The horizontal spectra are shifted upwards for better visibility.

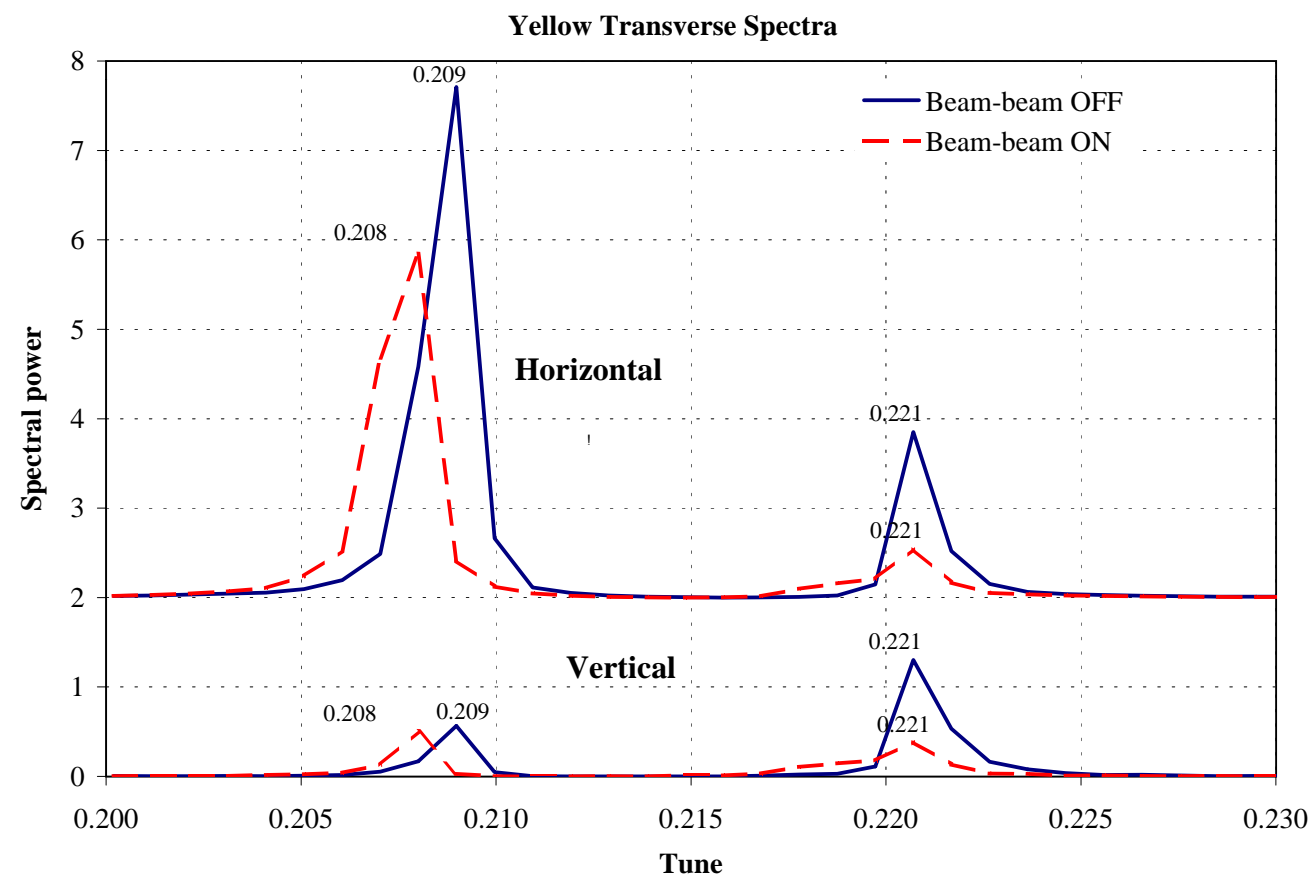

Figure 7: Yellow transverse spectra from 1024 consecutive turns after a small kick in operation. Two curves show the situation with the beam-beam interaction on, two with the beam-beam interaction off. The horizontal spectra are shifted upwards for better visibility. 


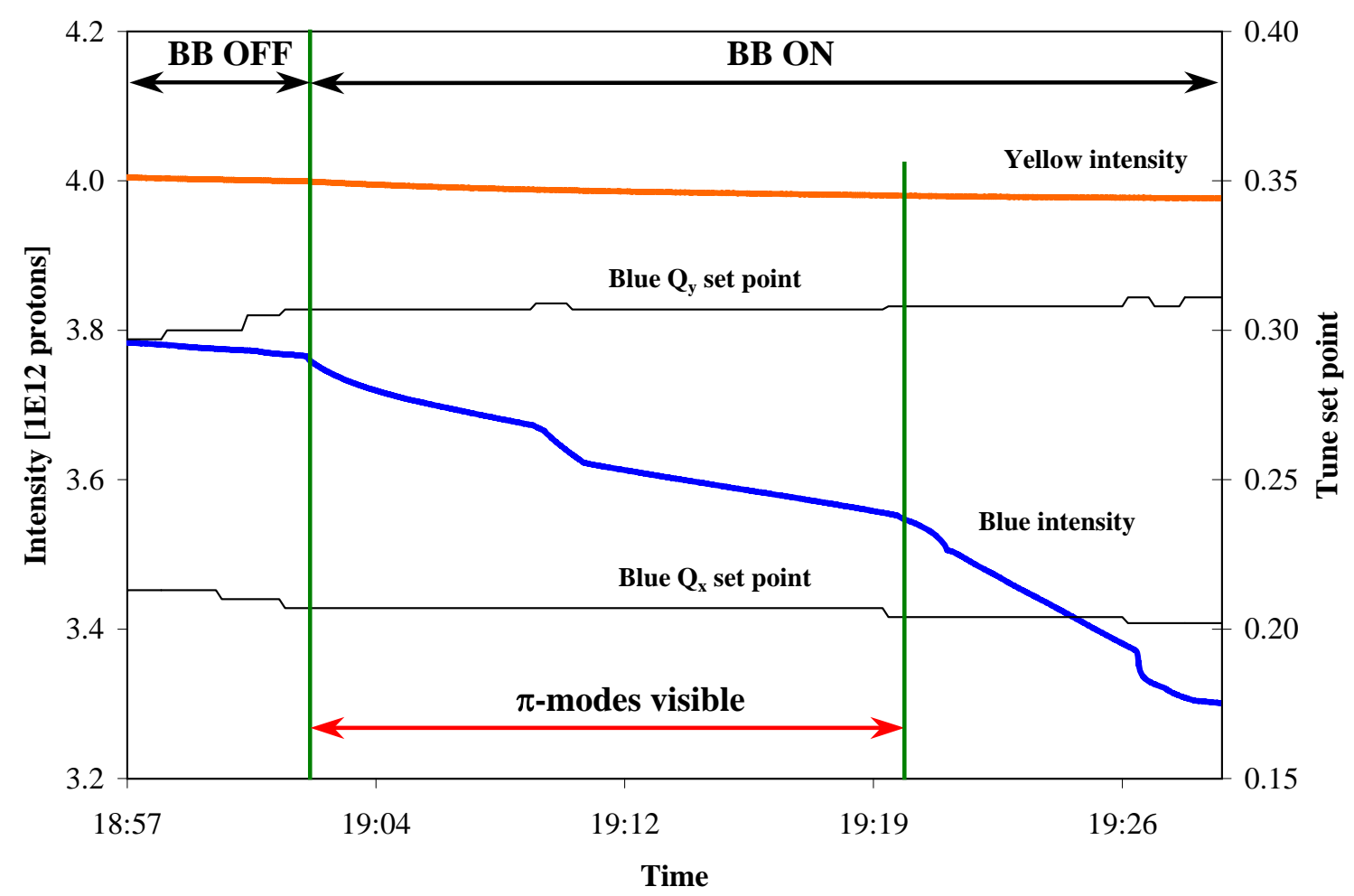

Figure 8: Blue and Yellow beam intensity and Blue tune set points during tuning for lifetime at the beginning of a proton store. $\pi$-modes were only visible for certain tune set points.

The $\pi$-modes appeared and disappeared as the Blue tunes were changed to improve the beam lifetime. This is shown in Fig. 8. After the beams were brought into collision, the Blue intensity dropped and $\pi$-modes became visible. It is not clear to what extent the existence of the $\pi$-modes contributed to the lifetime deterioration. Sharp reductions in the beam lifetime are also observed without the existence of $\pi$-modes. After the Blue horizontal tune set point was lowered by 0.003 and the Blue vertical tune set point raised by 0.001 , the $\pi$-modes disappeared again. Note that the vertical tune set point does not coincide with the measured tune. Changes in the set point are, however, close to measured tune changes. No increases in the transverse emittances were observed with the ionization profile monitor (IPM) during tuning. But only relatively large changes in the emittance are detectable.

\section{Summary}

Coherent beam-beam modes were observed in an experiment at RHIC with a beam-beam parameter $\xi=0.003$ and a single collision per turn. The measured difference between the $\sigma$ - and $\pi$-modes is consistent with a Yokoya factor of $Y \approx 1.3$. The locations of the $\pi$-modes could be reproduced in a strong-strong simulation [8]. $\pi$-modes were also 
observed in routine operation with a beam-beam parameter $\xi=0.0015$, four collisions per turn and strong linear coupling. The $\pi$-modes could be suppressed by small changes in the tune.

\section{Acknowledgements}

We are thankful for support from the operations group, and for discussions with Y. Alexahin, M. Brennan, M.A. Furman, W. Herr, J.-P. Koutchouk, S. Peggs, J. Shi, and F. Zimmermann.

\section{References}

[1] Y. Alexahin, "On the Landau Damping and Decoherence of Transverse Dipole Oscillations in Colliding Beams", Part. Accel.,V59, p. 43; CERN-SL-96-064 (AP) (1996).

[2] R. E. Meller and R. H. Siemann, IEEE Trans. Nucl. Sci., NS-28, No.3, pp.2431, (1981);

[3] K. Yokoya, Y. Funakoshi, E. Kikutani, H. Koiso, J. Urakawa, "Tune Shift of Coherent Beam-Beam Oscillations", p. 40, KEK Preprint 89-14 (1989).

[4] J.P. Koutchouk, "ISR Performance Report - Beam-Beam Instabilities I", CERN LEPISR-TH/JPK/LV/rh (1979).

[5] J.P. Koutchouk, "ISR Performance Report - Beam-Beam Instabilities II", CERN LEPISR-OP/JPK/svw (1982).

[6] J.P. Koutchouk, "ISR Performance Report - A Numerical Estimate of the Coherent Beam-Beam Effect in the ISR", CERN ISR-OP/JPK-bm (1982).

[7] J.P. Koutchouk, "ISR Performance Report - Beam-Beam Instabilities III and Last", CERN LEP-ISR-OP/JPK/cc (1984).

[8] M. Vogt, J. A. Ellison, W. Fischer, and T. Sen, "Simulations of Coherent Beam-Beam Modes at RHIC", proceedings of the 2002 European Particle Accelerator Conference, Paris, France (2001). 